\title{
Medical Student Neurophobia: A Review Of The Current Pandemic And Proposed Educational Solutions
}

\author{
Joshua A. Cuoco, MS \\ New York Institute of Technology College of Osteopathic Medicine, \\ New York U.S.A \\ URL:http://dx.doi.org/10.19044/ejes.v3no3a4
}

\begin{abstract}
Traditionally, the subject of neuroscience has been one of the most difficult courses for medical students in undergraduate medical education. Over the last few decades, a fear of neurology and the neurosciences, termed neurophobia, has presented among medical students around the world. Today, neurophobia has resulted in medical students not learning neurology and neurosciences to a sufficient extent to be able to apply this knowledge to patients presenting with neurologic symptomatology. To address the crisis at hand, medical educators have developed novel learning modalities to fight this chronic disease of medical students. In this brief review, I examine the educational literature regarding the current pandemic of neurophobia as well as analyze published novel educational modalities that may be used to diminish neurophobia among medical students.
\end{abstract}

Keywords: Neurophobia, neuroscience, neurology, medical education

\section{Introduction}

The term neurophobia was created over two decades ago to describe medical students' fear of neurology and the neurosciences (Jozefowicz 1994). Today, neurophobia has run rampant among medical students with studies confirming a significant fear of neurology and the neurosciences in the United States, China, United Kingdom, Ireland, Sri Lanka, and the Caribbean (Ramos et al. 2016, Zinchuk et al. 2010, Lukas et al. 2014, Pakpoor et al. 2014, Flanagan et al. 2007, Matthias et al. 2013, Youssef 2009). Worldwide emergence of this phenomenon has encouraged medical educators around the globe to develop potential solutions to this problem (Roze et al. 2016, Dao et al. 2015, Anwar et al. 2015, Hudson 2006). This manuscript will review the current educational literature addressing neurophobia in various countries around the world. Furthermore, this 
manuscript will analyze published novel learning modalities that seek to address and diminish neurophobia among medical students.

\section{United States}

In a study by Zinchuk (2010), questionnaires were administered to third and fourth year medical students at a United States medical school and to internal medicine residents affiliated with the particular medical school. The questionnaire examined the difficulty of eight clinical specialties, including neurology (Zinchuk et al. 2010). Respondents indicated that they felt neurology was the clinical specialty that they had the least amount of knowledge in $(\mathrm{p}<0.001)$ as well as neurology being the most difficult clinical rotation $(\mathrm{p}<0.001)$ (Zinchuk et al. 2010). Furthermore, medical students indicated that they had the least amount of confidence when confronted with patients presenting with neurological complaints (Zinchuk et al. 2010). Zinchuk (2010) concluded that both medical students and residents exhibited significant difficulty as well as a low degree of confidence when confronted with patients presenting with neurologic symptomatology.

\section{China}

An investigation by Lukas (2014) surveyed senior medical students at a medical school in Wuhan, China, on their perceptions regarding knowledge of clinical neurology. Collected data indicated that students' self-assessment of knowledge pertaining to neurology ranked low, but not the lowest rank possible (Lukas et al. 2014). Furthermore, a positive correlation was observed between diagnosing/managing neurological patients and likelihood of specializing in the field of neurology (Lukas et al. 2014).

\section{United Kingdom}

In an inquiry by Pakpoor (2014), an online survey was implemented to all United Kingdom medical schools. The data indicated that medical students found neurology to be significantly more difficult than other clinical specialties (Pakpoor et al. 2014). Moreover, medical students were the least comfortable with thinking of a neurological differential diagnosis relative to the other clinical specialties ( $\mathrm{p}<0.0001$ for neurology) (Pakpoor et al. 2014).

\section{Ireland}

In a study by Flanagan (2007), questionnaires were administered to medical students as well as physicians from various colleges and hospitals throughout Ireland. The questionnaire was administered in two parts. The first part addressed medical students' perceptions of knowledge in neurology and confidence in dealing with patients with neurologic symptoms (Flanagan et al. 2007). Data in the first section of the questionnaire indicated that the 
respondents felt that their knowledge in neurology was limited $(\mathrm{p}<0.005)$ after nephrology, geriatrics, and rheumatology (Flanagan et al. 2007). Furthermore, participants found neurology to be the most difficult clinical specialty over all other disciplines $(\mathrm{p}<0.001)$ (Flanagan et al. 2007). The second part of the questionnaire requested respondents to indicate the reason why neurology was perceived as a difficult clinical specialty (Flanagan et al. 2007). Data in the second section of the questionnaire indicated that the number one reason respondents felt neurology to be a difficult specialty was due to poor teachings of neurology (Flanagan et al. 2007).

\section{Sri Lanka}

An investigational study by Matthias (2013) evaluated medical students' perceptions of neurology, via questionnaires, from the University of Sri Jayewardenepura, in Sri Lanka. Medical students indicated that neurology was the least interesting clinical specialty (18.5\%), the most difficult specialty for the majority of medical students (50.0\%), and the specialty in which they had the least amount of confidence in dealing with patients presenting with neurologic symptomatology (i.e., headache, numbness of extremities, dizziness) (Matthias et al. 2013). Furthermore, the major reason as to 'why neurology was the most difficult clinical specialty' was determined to be the necessary understanding of neuroanatomy and the complex neurologic examination (Matthias et al. 2013).

\section{The Caribbean}

A cross-sectional study by Youssef (2009) surveyed senior medical students of the University of the West Indie, St. Augustine, Trinidad \& Tobago, in the Caribbean. Medical students were asked four questions: (i) "What is your current level of interest in the following medical specialties?"; (ii) "What is your current level of knowledge in the medical specialties?"; (iii) "Do you think the subject is easy or difficult?"; (iv) "Why do you think neurology is difficult?" (Youssef 2009). Data collected showed that medical students found neurology to be the most difficult clinical specialty as well as the specialty that students had the least amount of knowledge in $(\mathrm{p}<0.001)$ (Youssef 2009). The major contributor to difficulty with neurology was determined to be the requirement to understand neuroscience, which was closely followed by the complex neurologic examination (Youssef 2009).

\section{Recent Educational Solutions Addressing Neurophobia}

Given the well-documented phenomenon of neurophobia worldwide, medical educators have begun to bring forth potential solutions to the crisis at hand (Zinchuk et al. 2010, Lukas et al. 2014, Pakpoor et al. 2014, Flanagan et al. 2007, Matthias et al. 2013, Youssef 2009). In response to 
neurophobia among medical students, educators have initiated innovative simulation-based programs, applied neuroanatomy electives, team-based learning in neuroscience blocks, and case-based teaching within the neuroscience block of the medical curriculum (Roze et al. 2016, Dao et al. 2015, Anwar et al. 2015, Hudson 2006).

A proposed learning modality by Roze (2016) to improve the understanding of neurological semiology was to initiate simulation training. A simulation-training program known as 'The Move' was implemented for third year medical students of Pierre and Marie Curie University in Paris, France, throughout the students' rotation in neurology (Roze et al. 2016). Using only mime gestures, medical students were taught to role-play patients presenting with neurologic syndromes such as parkinsonian, vestibular, pyramidal, and cerebellar (Roze et al. 2016). Furthermore, tonic-clonic seizures, axonopathies, and chorea were also taught and mimed by students (Roze et al. 2016). Questionnaires were administered to the students and teachers investigating the impact of the program (Roze et al. 2016). Students indicated that The Move program increased their motivation to understand neurologic semiology (78\%) as well as improved both their long-term memorization of the neurologic syndromes (86\%) and understanding of neurology (77\%) (Roze et al. 2016). Moreover, 77\% of students thought the skills learned in The Move would be useful for their clinical practice in the future (Roze et al. 2016). Importantly, 87\% of students and 95\% of teachers thought that it would be beneficial to include The Move program in their medical curriculum (Roze et al. 2016).

Granted that a cause of neurophobia is a poor understanding of neuroanatomy, Dao (2015) examined the efficacy in implementing an applied neuroanatomy elective dependent upon students utilizing interactive and artistic activities. Offered to first-year medical students, the elective in neuroanatomy consisted of four sessions encompassing four neurosensory modalities including taste/olfaction, auditory, vision, and proprioception/balance (Dao et al. 2015). Each module was 2-hours in length consisting of a 15 minute lecture component and 105 minute laboratory component (Dao et al. 2015). The lecture component was comprised of a review of the neurosensory pathways, clinical vignettes pertaining to neurology, and an introduction to the laboratory component (Dao et al. 2015). The laboratory component consisted of specific activities targeted to enforce the neurosensory pathways taught in the previous lecture component: (i) taste/olfaction module integrated foods that highlighted each of the five taste sensations; (ii) auditory module integrated beat-matching music, DJing, and creating new sounds with use of synthesizers; (iii) visual module integrated photography as well as experiencing touch alone with use of blindfolding; (iv) proprioception/balance module integrated balance with 
unicycling, balance boarding, slack lining, and yoga (Dao et al. 2015). Data collected pre- and post-course indicated that the medical students were significantly more familiar as well as comfortable with the taste/olfaction, auditory, vision, and proprioception/balance pathways that were taught to them (Dao et al. 2015).

Recently, team-based learning has increasingly been regarded by undergraduate medical schools as an effective teaching modality to assist students in understanding lecture material (Anwar et al. 2015). In a study by Anwar (2015), team-based learning was implemented throughout a thirdyear neuroscience block. Medical educators evaluated the effect of teambased learning on student learning and correlated it with the medical student's academic performance in the summary exam (Anwar et al. 2015). Data showed that students who attended the team-based learning modules performed better on the summary exam than those who did not attend teambased learning modules (Anwar et al. 2015).

A case based teaching modality was developed and implemented at Adelaide University in the Department of Physiology to integrate neuroscience with clinical neurology in the undergraduate medical curriculum (Hudson 2006). Each medical student attended a total of four 2hour case based teaching exercises: (i) sensory function module teaching the interpretation of findings in the patient history; (ii) motor function module teaching the interpretation of motor and sensory physical examination findings; (iii) a combined module on cranial nerve function and radiological imaging teaching the interpretation of cranial findings and utilizing radiological imaging to confirm a diagnosis, respectively; (iv) higher function module teaching how to present patients with neurologic symptomatology (Hudson 2006). Data from the case based learning initiative was gathered via questionnaires pertaining to the efficacy of the learning modality (Hudson 2006). Overall, students reported that case based learning was effective in facilitating an integration of clinical neurology with the neurosciences (Hudson 2006). Some students admitted to neurophobia, which was alleviated by the initiative to a degree; however, these students requested additional case based learning modules to further reduce their neurophobia (Hudson 2006).

\section{Conclusion}

A chronic disease of medical students, neurophobia has been reported to affect students around the world. Novel initiatives are essential not only to cure those currently affected but also to prevent the next generation of physicians from developing a fear for neurology and the neurosciences. 


\section{References:}

Jozefowicz, R.F. (1994). Neurophobia: the fear of neurology among medical students. Archives of Neurology, 51:328-329.

Ramos, R.L., Cuoco, J.A., Guercio, E., Levitan, T. (2016). Quantitative description of osteopathic medical student interest in neurology and psychiatry. The Journal of the American Osteopathic Association, 116(7):462-471.

Zinchuk, A.V., Flanagan, E.P., Tubridy, N.J., Miller, W.A., and McCullough, L.D. (2010). Attitudes of US medical trainees towards neurology education: "Neurophobia" - a global issue. British Medical Council Medical Education, 10:49.

Lukas, R.V., Cooper, B., Morgan, I., Brorson, J.R., Dong, H., and Sherer, R. (2014). Attitudes toward neurosciences in medical students in Wuhan, China: a survey study. World Neurosurgery, 82(3-4):266-269.

Pakpoor, J., Handel, A.E., Distanto, G., Davenport, R.J., Giovannoni, G., Ramagopalan, S.V., and on behalf of the Association of British Neurologists. (2014). National survey of UK medical students on the perception of neurology. British Medical Council Medical Education, 14:225.

Flanagan, E., Walsh, C., and Tubridy, N. (2007). 'Neurophobia' - attitudes of medical students and doctors in Ireland to neurological teaching. European Journal of Neurology, 14:1109-1112.

Matthias, A.T., Nagasingha, P., Ranasinghe, P., and Gunatilake, S.B. (2013). Neurophobia among medical students and non-specialist doctors in Sri Lanka. British Medical Council Medical Education, 13:164.

Youssef, F.F. (2009). Neurophobia and its implications: evidence from a Caribbean medical school. British Medical Council Medical Education, 9:39. Roze, E., Flamand-Roze, C., Meneret, A., Ruiz, M., Le Liepure, H., Duguet, A., Renaud, M.C., Alamowitch, S., and Steichen, O. (2016). 'The Move', an innovative simulation-based medical education program using roleplay to teach neurological semiology: students' and teachers' perceptions. Neurological Review172:289-294.

Dao, V., Yeh, P.H., Vogel, K.S., and Moore, C.M. (2015). Applied neuroanatomy elective to reinforce and promote engagement with neurosensory pathways using interactive and artistic activities. Anatomical Science Education, 8:166-174.

Anwar, K., Shaikh, A.A., Sajid, M.R., Cahusac, P., Alarifi, N.A., and Shedoukhy, A.A. (2015). Tackling student neurophobia in neurosciences block with team-based learning. Medical Education Online, 20:28461.

Hudson, J.N. (2006). Linking neuroscience theory to practice to help overcome student fear of neurology. Medical Teacher, 28(7):651-653. 